\title{
Using the conservative nature of fresh leaf surface density to measure foliar area**
}

\author{
Omar S. Castillo ${ }^{1}$, Esther M. Zaragoza ${ }^{1}$, Carlos J. Alvarado ${ }^{1}$, Maria G. Barrera ${ }^{1}$, \\ and Nabanita Dasgupta-Schubert ${ }^{1,2} *$ \\ ${ }^{1}$ Institute of Chemical Biology (IIQB), University of Michoacan (UMSNH), Cd. Universitaria Morelia, Michoacan 58060, México \\ ${ }^{2}$ Department of Environmental Biotechnology, Helmholtz Centre for Environmental Research (UFZ), \\ Permoserstraße 15, 04318 Leipzig, Germany
}

Received November 19, 2013; accepted May 8, 2014

\begin{abstract}
A b s tra ct. For a herbaceous species, the inverse of the fresh leaf surface density, the Hughes constant, is nearly conserved. We apply the Hughes constant to develop an absolute method of leafarea measurement that requires no regression fits, prior calibrations or oven-drying. The Hughes constant was determined in situ using a known geometry and weights of a sub-set obtained from the fresh leaves whose areas are desired. Subsequently, the leaf-areas (at any desired stratification level), were derived by utilizing the Hughes constant and the masses of the fresh leaves. The proof of concept was established for leaf-discs of the plants Mandevilla splendens and Spathiphyllum wallisii. The conservativeness of the Hughes constant over individual leaf-zones and different leaftypes from the leaves of each species was quantitatively validated. Using the globally averaged Hughes constant for each species, the leaf-area of these and additional co-species plants, were obtained. The leaf-area-measurement-by-mass was cross-checked with standard digital image analysis. There were no statistically significant differences between the leaf-area-measurement-by-mass and the digital image analysis measured leaf-areas and the linear correlation between the two methods was very good. Leaf-areameasurement-by-mass was found to be rapid and simple with accuracies comparable to the digital image analysis method. The greatly reduced cost of leaf-area-measurement-by-mass could be beneficial for small agri-businesses in developing countries.

K e y w o r d s: leaf area, leaf mass, planimetry
\end{abstract}

\section{INTRODUCTION}

Leaves are of fundamental importance to plants. They constitute the plant power generation and aerial environmental sensing units. The amount of photosynthetic light

*Corresponding author e-mail: nita@ifm.umich.mx

**This work was financed by CONACyT of Mexico for NDS through the fellowship No. UMSNH-173076 for her sabbatical year 2012 at the UFZ, Germany. harvested depends directly on the leaf-area (LA), which affects plant growth and bio-productivity and hence also the agro-economic return from the crop. Additionally, leaf biometrics are markers of nutritional status or environmental stress on the plant (Meziane and Shipley, 1999; Vile et al., 2005). The leaf area index (LAI) of a stand of plants is one of the most frequently used parameters for the analysis of canopy structure and is an important structural characteristic of crop/forest monitoring and crop productivity (Behera et al., 2010). It is fundamentally important as a parameter in land-surface processes and for parametrizations in climate models. Direct methods of LAI determination are based on the destructive technique of the LA measurements of the individual leaves and are considered to be the most accurate, albeit time-consuming, ways of determining LAI (Jonckheere et al., 2004). Nonetheless, they are used to benchmark nondestructive methods of LAI determination (Behera et al., 2010; Kirk et al., 2009).

Hence the measurement of LA occupies an important place in the slew of plant biometric techniques. This importance has stimulated the proposition of a variety of methods for LA measurement (Coombs et al., 1985; Igathinathane et al., 2006; Korva and Forbes, 1997; Ma et al., 1992; Mohsenin, 1986; O'Neal et al., 2002; Rico-Garcia et al., 2009; Sharatt and Baker, 1986; Singh et al., 1995; for a review of LAI determination, see Jonckheere et al., 2004). The method that has emerged as the most frequently used, particularly for herbaceous broad-leaved species, and which has been commercialized, is planimetry (Jonckheere et al., 2004): the leaf, excised at its junction to the petiole, is laid on a scannertype bed, and the pixel count of its digital image using 
appropriate software, is used to quantify its area (O'Neal et al., 2002 and references therein, LI-3000, Li-Corr, Lincoln, NE, USA). While this method, henceforth called digital image analysis (DIA), is well-established, some difficulties persist. Leaves with non- planar laminae (wavy leaves) do not sit flush against the bed so that the kinks could cause zones to be occulted; the pixel contrast for leaves with insufficient chlorophyll content (yellowish leaves) is sometimes not picked up accurately enough by the software. Both can result in an underestimation of the area (Rico-Garcia et al., 2009; Tsuda, 1999). The problems can be corrected to some extent by positioning and flattening the leaves manually and by manual grey-scale corrections of the digitized image, but both detract from the speed of the procedure. The central motivation of this work was to find an accurate, cost-effective, non-DIA technique for determining leaf areas using the functional traits of leaves in an absolute as against the relative way ie without the use of prior statistically fitted calibrations.

In the context of a relative determination, it is germane to briefly recapitulate the second traditionally used method of LA determination - the gravimetric method. The method consists of first determining the fresh LAs of a sub-sample of leaves of a particular species drawn from the global field sample using a DIA method and correlating these with their dry masses. Then the LA to dry leaf mass ratio or specific leaf area (SLA), is applied to the dry masses of all the harvested leaves to obtain their areas. The method is not stand-alone (ie it requires a prior instrument of LA determination), it involves extra processing (oven-drying) and the SLA is not a well-conserved quantity (see below) in that it has been observed to possess wide spatial and temporal variations in many tree species (Fila and Sartorato, 2011; Jonkheere et al., 2004). However it is a convenient (more rapid) method for the estimation of LAI for very numerous leaf samples (Jonckheere et al., 2004).

Roderick and Cochrane (2002) reported the test and corroboration of the observation made about three decades earlier by Hughes et al. (1970). Their combined measurements covered a large number of plant species. They observed that for herbaceous species there apparently exists a unique species-specific relationship between the area and the mass of fresh leaves.

The relationship connecting the leaf area (projected normal to the surface), $A\left(\mathrm{~m}^{2}\right)$ and the fresh leaf mass $M(\mathrm{~kg})$ is:

$$
A=\mathrm{K} M \text {, }
$$

where $\mathrm{K}$ termed the Hughes constant by Roderick and Cochrane (2002), is related to the density $(\rho)$ and thickness $(\tau)$ of the fresh leaf as:

$$
\mathrm{K}=\frac{1}{\rho \tau} .
$$

Since the dimension of $\mathrm{K}$ is $\left(\mathrm{m}^{2} \mathrm{~kg}^{-1}\right)$, it can be construed as the fresh leaf specific area, or in analogy with thickfilm materials technology, the inverse of the fresh leaf surface density.
Using measurements of the LA and leaf mass from 15 broad-leaved species Roderick and Cochrane (2002) showed that for each species K was approximately a constant despite variations in leaf thickness and water content. The latter would significantly affect $\rho$. Based on these results, they contend that for a given species, the Hughes constant is likely to be much more conservative than other functional attributes, eg leaf area per unit of dry mass (the specific leaf area, SLA), leaf water content, etc. This is a remarkable finding. It implies that despite factors affecting $\rho$ and $\tau$ separately, the plant dynamically adjusts both through their functional connectedness, to maintain $\mathrm{K}$ as constant.

The utility of conserved quantities is that they allow accurate measurements to be made. The analogy to the leaf in materials science is the thick self-supporting film whose density is constant and known. The thickness of the film can then be determined accurately by punching/cutting out a geometrical shape of known area, weighing it and thereupon utilizing Eqs (1) and (2). Using the property of the conservativeness of $\mathrm{K}$ and an approach inspired by thick-film technology, we now proceed to describe the development of the leaf-area-measurement-by-mass (LAMM) method of LA measurement.

The aim of the present study is to elucidate the LAMM technique for the determination of the foliar area of herbaceous plants. This aim is realized in the three stages of - the checks on the conservativeness of K over differently sampled areas of the given leaf and for different leaf types; - the estimation of the accuracy of the method;

- the determination of the areas of leaves of a different individual of the same species using the $\mathrm{K}$ value previously determined from a another plant, which tests the constancy of $\mathrm{K}$ for the given species.

\section{MATERIALS AND METHODS}

Plants of two different species, Mandevilla splendens, common name Pink-allamanda and Spathiphyllum wallisii, common name Peace-lily, were used for the exposition of the LAMM method. At every stage, measurements by DIA on the same specimens were made to benchmark the LAMM against this standard method.

Let us suppose that there are $v$ broad-leaved plants of a particular species in an experimental set, whose total foliar area (FA) is desired to be measured. The index $j$ for the individual plants runs from 1 to $v(1 \leq j \leq v)$. Further, each plant has $n$ leaves. The Hughes constant for the $i$ th leaf of any plant where $1 \leq i \leq n$ is:

$$
\mathrm{K}_{i}=\frac{1}{\rho_{i} \tau_{i}} .
$$

The $i$ th leaf is detached at its base from the petiole and weighed, to yield the mass $M_{i}$. Now, using a sharp-edged die-punch of known ID, discs are punched out in a random manner over the face of the leaf, taking care to see that ribbed and fleshy areas are equally sampled. In the general 
case, several die-punches of differing ID may be used. The punch-out process will result in a maximum of $P$ discs where the ID of the $p$ th $\operatorname{disc}(1 \leq \mathrm{p} \leq P)$ is $d_{p}$ and its mass as measured in the weighing balance is $m_{p}$. Its area $a_{p}$ then is:

$$
a_{p}=\pi\left(\frac{d_{p}}{2}\right)=\mathrm{K}_{i} m_{p},
$$

which can be easily calculated.

If all the $P$ discs are pooled together and weighed to yield the mass $m$ :

$$
m=\sum_{p=1}^{p=P} m_{p}=\left(\frac{1}{K_{i}}\right) \sum_{p=1}^{p=P} a_{p} .
$$

Equations (4) and (5) assume that the Hughes constant is invariant over the face of the leaf and has the single value $K_{i}$, making it applicable for all the discs. This assumption will be tested as a part of the experimental procedure described further on.

The area of the $i$ th leaf is then:

$$
A_{i}=\mathrm{K}_{i} M_{i} .
$$

Doing likewise for all the $\mathrm{n}$ leaves of the plant we will obtain their different values of $\mathrm{K}$ and their different areas. We will then obtain the total FA of the plant, $A$, by summing over all the different areas $A_{i}$ :

$$
A=\sum_{i=1}^{i=n} A_{i}=\sum_{i=1}^{i=n} \mathrm{~K}_{i} M_{i}
$$

Roderick and Cochrane (2002) pointed out that the Hughes constant is nearly invariant with respect to leaf type in the given plant - an observation that we corroborate in our experimental procedure.

Hence,

$$
\mathrm{K}_{1}=\mathrm{K}_{2}=\ldots=\mathrm{K}_{\mathrm{i}} \ldots=\mathrm{K}_{\mathrm{n}}=\mathrm{K}_{\mathrm{G}} .
$$

Thus a fine-tuned stratification of the value of the Hughes constant leaf by leaf, becomes unnecessary and the procedure can be shortened. One would only need to sample a much smaller number of arbitrarily selected leaves of the plant, punch out the discs from these as stated above, weigh them and obtain the value of $\mathrm{K}$ which is now the Hughes constant representing the whole plant. We call it the global average $\mathrm{K}\left(K_{G}\right)$. Alternatively, a value for $K_{G}$ may be derived as the average of the measured values of $\mathrm{K}$ corresponding to arbitrarily selected sub-sets of different leaf types (young, old, etc.).

Then,

$A=\sum_{i=1}^{i=n} A_{\mathrm{i}}=\sum_{i=1}^{i=n} \mathrm{~K}_{\mathrm{i}} M_{i}=\sum_{i=1}^{i=n} \mathrm{~K} M_{i}=\mathrm{K} \sum_{i=1}^{i=n} M_{i}=K M=\mathrm{K}_{\mathrm{G}} M$, where $M$ is the weight of all the detached and intact leaves of the plant, obtained by weighing prior to the punch-out of discs from some of them.

For the laboratory experiment that consists of the $v$ number of plants, the area $A$ in Eq. (9) is actually the FA of the $j$ th plant of the set, $A_{j}$ that has the total leaf mass $M_{j}$ and the Hughes constant $\mathrm{K}_{\mathrm{Gj}}$. The FA of all the $v$ plants of the set taken as a single entity, $A$, is then the sum over all $A_{j}$ :

$$
A=\sum_{j=1}^{j=v} A_{j}=\sum_{i=1}^{j=v} \mathrm{~K}_{\mathrm{Gj}} M_{j}
$$

Roderick and Cochrane (2002) observe that the Hughes constant remains approximately the same for all plants of the same species (but varies between species). In that case, stratification on a plant by plant basis to obtain the value of $K_{G j}$ for each is not required and the procedure can be further simplified. The overall global value of $\mathrm{K}$ for all the plants in the set, $K_{G}$, can be obtained by punching out discs from randomly selected leaves of different plants in the set using die-punches of known IDs in the manner described before. Prior to the punch-out operation, all the detached leaves of all $v$ plants are pooled and weighed to yield the mass $M$. Therefore, the total FA of all $v$ plants becomes simply:

$$
A=\sum_{j=1}^{j=v} \mathrm{~K}_{\mathrm{Gj}} M_{j}=\mathrm{K}_{\mathrm{G}} \sum_{j=1}^{j=v} M_{j}=\mathrm{K}_{\mathrm{G}} M
$$

The process is simple and rapid. In contrast, for a typical DIA measurement, the detached leaves have to be placed carefully on the scanner-bed which itself might be space limited to only a certain number of leaves at a time and furthermore, the measurement result depends on the analysis of the image of each leaf. This is the likely reason why for large sample sizes, the DIA is not considered to be the most efficient LA measurement technique and the gravimetric method, in spite of its deficiencies, is considered as a viable option (Jonckheere et al., 2004).

The experimental procedure is now described as follows.

Two nursery grown plants, one a four month old $M$. splendens and the other a two year old $S$. wallisii were chosen for the experimental investigation of LAMM. The leaves selected were 6 of $M$. splendens that included the morphological types of 1 mature-chlorotic leaf, 2 mature and 3 young leaves, and 5 of $S$. wallissi that were all mature. Stainless steel die-punches of IDs 1.3, 1.0 and $0.5 \mathrm{~cm}$ (Leon Weill S.A., Mexico) were used to punch out several discs from these leaves. The masses of the all the discs of a particular diameter for each leaf type from the two plants were obtained by weighing the discs on a laboratory balance (Ohaus AdventurerTM SL, Ohaus Corp., USA). Now utilizing Eq. (4), the K values for all the selected leaves of each plant were separately obtained for discs corresponding to 
T a b l e 1. Values of fresh leaf surface density, $\left(\mathrm{K}^{-1}\right)$ obtained for the leaves of the plants $M$. splendens and $S$. wallisii with respect to the diameters, of the punched-out leaf discs used to obtain $\mathrm{K}^{-1}$

\begin{tabular}{|c|c|c|c|c|c|c|c|}
\hline \multirow{3}{*}{ Leaf type } & \multicolumn{4}{|c|}{$\mathrm{K}^{-1} 10^{2}\left(\mathrm{~kg} \mathrm{~m}^{-2}\right)$} & \multirow{3}{*}{$\left(\mathrm{K}^{-1}\right)_{\text {avg }} 10^{2}$} & \multirow{3}{*}{$\left(\mathrm{K}^{-1}\right)_{G} 10^{2}$} & \multirow{3}{*}{$\mathrm{CV}(\%)$} \\
\hline & 1.3 & 1.0 & 0.5 & 0.3 & & & \\
\hline & \multicolumn{4}{|c|}{$(\mathrm{cm})$} & & & \\
\hline \multicolumn{8}{|c|}{ Mandevilla splendens } \\
\hline L1-MC & 3.16 & 2.55 & 3.06 & 2.67 & $(2.86 \pm 0.30)^{*}$ & - & 10.4 \\
\hline L2-M, L3-M & $2.89 \pm 0.25$ & $2.67 \pm 0.23$ & $3.06 \pm 0.01$ & $2.54 \pm 0.16$ & $(2.79 \pm 0.23)^{*}$ & - & 8.2 \\
\hline \multirow[t]{2}{*}{ L4-Y to L6-Y } & $2.84 \pm 0.45$ & $2.57 \pm 0.50$ & $2.73 \pm 0.43$ & $2.36 \pm 0.47$ & $(2.62 \pm 0.45)^{*}$ & - & 17.1 \\
\hline & & & & & & $(2.72 \pm 0.30)^{* *}$ & 11.2 \\
\hline \multicolumn{8}{|c|}{ Spathiphyllum wallisii } \\
\hline \multirow[t]{2}{*}{ L1-M to L5-M } & $2.15 \pm 0.23$ & $2.14 \pm 0.43$ & $2.10 \pm 0.28$ & $1.72 \pm 0.19$ & $(2.03 \pm 0.25)$ & & \\
\hline & & & & & & $(2.03 \pm 0.25)^{* *}$ & 12.3 \\
\hline
\end{tabular}

L1-L6 - leaves labelled by numbers, leaf types: $\mathrm{MC}$ - mature with chlorosis, $\mathrm{M}$ - mature without chlorosis, $\mathrm{Y}$ - young. $\left(\mathrm{K}^{-1}\right)_{\text {avg }}$ and $\left(\mathrm{K}^{-1}\right)_{\mathrm{G}}$ are respectively: the averages for each leaf-type over all the disc sizes, and the global average over all disc sizes and leaf-types for each plant species. CV (\%) is the coefficient of variation for the various average values. Statistically *not significant, **significant, differences computed by the t-test.

each die-punch ID. The K averages for each size (diameter) for each leaf morphological-type as well as the averages over all disc diameters for each leaf type were then computed. Finally the $\mathrm{K}_{\mathrm{G}}$ value was computed as the grand average over all disc diameters for all selected leaves of the given plant.

Prior to punching out the leaf discs, the areas of the 6 and 5 leaves respectively of $M$. splendens and $S$. wallisii were measured by the DIA method as specified by O'Neal et al. (2002). This method had been corroborated by the use of the LI-3000 commercial leaf area meter. The leaves were placed on the bed of the scanner (Canon PIXMA MP520) under a blue paper background that was graduated along the $\mathrm{X}-\mathrm{Y}$ axes to the maximum length of $15 \mathrm{~cm}$. The colour photographs of 300 ppi resolution were exported to the desktop of a standard PC in the imaging format JPEG as required by the image analysis software. This software was Sidelook (Nobis and Hunziker, 2005; Zehm et al., 2003). It was used in the manner described in the software manual (www.appleco. ch/sidelook_sample.zip) to analyse the images and obtain their areas. Additionally, the software Image- $J^{\mathbb{R}}$ was also used where the protocol as stated by its on-line manual (http:// rsbweb.nih.gov/ij/docs/menus/analyze.html) was followed to obtain the leaf areas. Later, the areas of the punched out leaf discs were also measured using the same softwares.

For each plant, the $\mathrm{K}_{\mathrm{G}}$ values were used to obtain the areas of the discs of the different sizes (diameters) for the leaves of the different morphological types and the average (corresponding to each diameter) was obtained. Only in the case of $M$. splendens the areas for discs from the mature- chlorotic leaf were excluded because comparison with the DIA was not possible due to Image-J producing an aberrant result. Since the geometrical area for each diameter is known, the accuracy of the LAMM and the DIA methods were obtained by comparing the absolute geometrical areas of the discs with the average LAMM derived and the average DIA derived areas.

Now, utlizing the $\mathrm{K}_{\mathrm{G}}$ values, the area of each of the selected leaves of the two plants (including the maturechlorotic one of $M$. splendens), was obtained by LAMM and compared to the areas obtained by the DIA methods.

To determine the time factor involved in each type of measurement, the duration of measurement of the LAs using the LAMM and the DIA (SIDELOOK) methods were timed. Finally, from two different individuals of the same plant species, $M$. splendens and $S$. wallisii, 9 leaves of the former and 5 of the latter were randomly selected. One of the 9 leaves of $M$. splendens was chlorotic. Using the $\mathrm{K}_{\mathrm{G}}$ values obtained from the same plant types of the previous set, the area of each of the currently selected leaves was obtained by the LAMM technique. Their areas had already been obtained by the DIA method. The results using each technique were compared.

For all measurements, the errors on primary determinations were computed as the standard errors, and for the determination of derived quantities, the standard formulae for error propagation were used (Mandel, 1984). Since the DIA measurement on each leaf as a single entity was a unique measurement, no statistical error was assigned to it. 
The Eqs (12) and (13) were used to compute the \% error $(E)$ or relative deviation $(R D)$ of the experimental quantity $(X)$ with respect to the reference $(R)$, and the \% accuracy $(A)$ (Mandel, 1984).

$$
\begin{gathered}
E \text { or } R D(\%)=\left|\frac{R-X}{R}\right| 100, \\
A(\%)=100-E(\%) .
\end{gathered}
$$

Furthermore, to determine whether the results were significantly different statistically or not between two sets of measurements (intra-LAMM or inter-LAMM and DIA), the Student t-test was applied (Mandel, 1984).

\section{RESULTS AND DISCUSSION}

The results for the determination of $\mathrm{K}$ from various sized discs obtained from leaves of different type from $M$. splendens and $S$. wallisii are summarised in Table 1. The values for the inverse of $\mathrm{K}$ are shown because $1 / \mathrm{K}$ has the dimension of $\left[\mathrm{ML}^{-2}\right]$ which is the way the surface density of materials in thick-film technology is described. Our first objective was to see whether in the case of real leaves the value of the Hughes constant varies across the face of the leaf $i e$ with respect to the size of the zone sampled. The coefficient of variation $(\mathrm{CV})$ is an indication of the random variability of the quantity under review, in other words its precision (Mandel, 1984). We see that the variability across the size of the zone sampled is not very different between the mature and mature-chlorotic leaves of $M$. splendens (8.2 and $10.4 \%$ ) but the variability is almost doubled for the young leaves $(17.1 \%)$ which suggests that for these developing leaves the Hughes constant is much more of a fluctuating quantity over the leaf face even while maintaining its average value close to those for other leaf types. The CVs for the global average surface densities for both plant species are close, in the environs of $11-12 \%$. The CV for the global average surface density of a light-green coloured plasticfilm wrapped card-paper sheet treated as a simulator of a leaf (results not shown), was $6.24 \%$. The variability for real leaves is therefore, only about double that of an industrially fabricated sheet where every attempt is made to keep the surface density constant. It shows the remarkable degree of regulation by plants in maintaining the uniformity of surface density (or its inverse, the Hughes constant) despite differences in leaf morphology, age, and state of health.

Our second objective was to see if the Hughes constant varies with leaf type and if it varies between plant species. From Table 1 we note that between the average values of $1 / \mathrm{K}$ (indicated by $\left(\mathrm{K}^{-1}\right)_{\mathrm{avg}}$ ) for the three leaf types in $M$. splendens there exists no statistically significant difference. However, the global average value of $1 / \mathrm{K}\left(\left(\mathrm{K}^{-1}\right)_{\mathrm{G}}\right.$, Table 1$)$ does differ statistically significantly between the two plant species. Both these results serve as quantitative corroboration of the observations made earlier by Roderick and Cochrane (2002) viz. that the Hughes constant is robustly conserved within a species but it varies between species.

Figure 1 shows the average areas of the different sized discs obtained from the mature and young leaves of $M$. splendens, where for the LAMM determinations, the $\mathrm{K}_{\mathrm{G}}$ value had been used. The mature chlorotic leaf had been omitted because Image-J produced aberrant results for it. This was possibly because the areas with insufficient chlorophyll resulted in spurious white pixels that produced erroneous results for the area. This effect has also been noted by Tsuda (1999) when using an automatic leaf area meter that uses a DIA based technique. The accuracies for the two methods for all the discs, lay between $95-96 \%$ for the LAMM and $96-99 \%$ for the DIA. Figure 2 shows the same type of determinations for the mature leaves of $S$. wallisii.

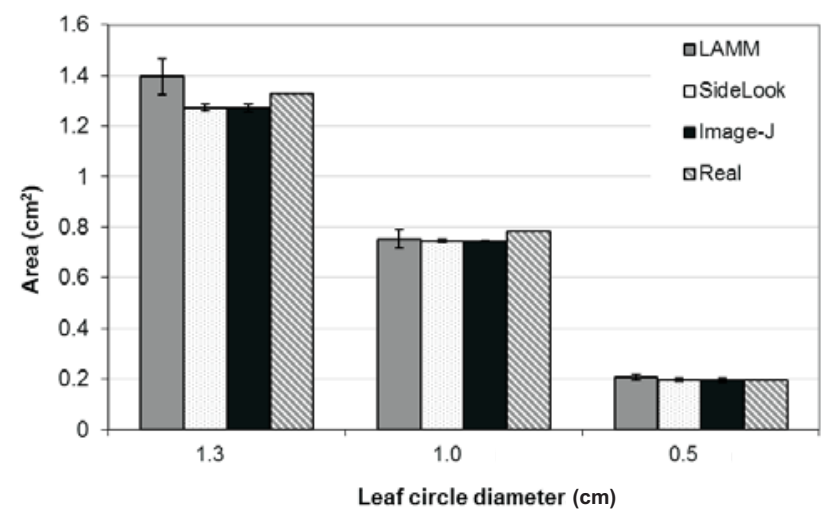

Fig. 1. Comparison of the areas of leaf discs of diameters 1.3, 1.0 and $0.5 \mathrm{~cm}$ punched out from 2 mature (excluding the chlorotic) and 3 young leaves of $M$. splendens (Table 1) measured by the LAMM technique and the DIA using SideLook (SL) and Image-J (IJ). The LAMM areas are the averages of all discs of the given diameter obtained using $\mathrm{K}_{\mathrm{G}}$. The DIA results pertain to the same discs as were used for the LAMM and are the averages over all discs of the given diameter. The accuracies of (a) the LAMM and (b) the DIA with SL and IJ, over the 3 disc sizes lay in the ranges of (a) $95-96 \%$ and (b) $96-99 \%$.

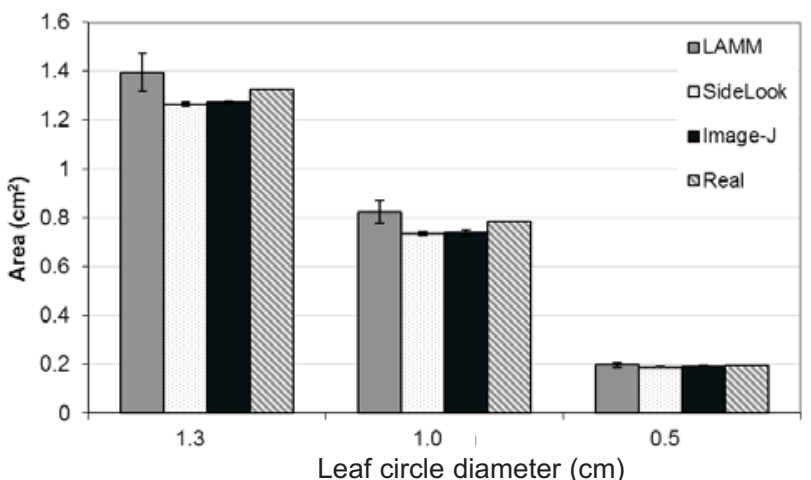

Fig. 2. Comparison of areas of leaf discs of the three diameters obtained from 5 mature leaves of $S$. wallisii (Table 1). The accuracies of (a) the LAMM and (b) the DIA methods using SL and IJ over the 3 disc sizes lay in the ranges of (a) $95-99 \%$ and (b) 94-97\% respectively. 
Again, the $\mathrm{K}_{\mathrm{G}}$ value had been used for the LAMM measurements. For these leaves the accuracies obtained by the LAMM and by the DIA were 95-99 and 94-97\%, respectively.

We note therefore, that for geometrical leaf areas obtained from both plant species, the LAMM and DIA accuracies are similar. Thus the LAMM technique proves to be at least as accurate as the standard technique of DIA. As far as the time efficiency of the two types of measurement is concerned, each SIDELOOK-DIA measurement took about 1-1.5 min to complete when no manual interventions for image corrections were utilised. The LAMM took on average about half this time. Hence the LAMM is also a rapid technique.

Figure 3 shows the areas of the 6 leaves of M. splendens (L1 to L6 with Y and M standing for 'young' and 'mature') obtained by LAMM using the $K_{G}$ value and by DIA. The mature-chlorotic (MC) leaf has also been included. The Image-J result for it clearly appears to be an underestimate. The average relative deviation between the LAMM and the SIDELOOK measured areas with the latter taken as the reference, was $10.4 \pm 5.7 \%$. For real leaves of irregular shape the accuracies for the two methods cannot be quanti- tatively ascertained against the calculated geometrical area as was done for the leaf discs. One can only determine whether the area values are statistically close or not. The ap- plication of the Student t-test to the overall average values of the areas obtained from the LAMM and the DIA indicated no statistically significant difference $(\mathrm{p}<0.05)$ between the two.

Figure 4 shows the analogous results for the 5 mature leaves of $S$. wallisii. For this plant the average relative deviation between the LAMM and SIDELOOK measured areas was $9.3 \pm 4.9 \%$. As in the case of M. splendens, there was no statistically significant difference $(p<0.05)$ between the overall average of the leaf areas obtained by the LAMM and by the DIA.

For both plants we notice that:

- the Image-J and the SIDELOOK (barring the case for the chlorotic leaf) produce identical results and that

- these in general are lower than the corresponding LAMM values.

The first observation indicates that the algorithms used in both softwares perform the same operations, but since the version of SIDELOOK used here allowed a manual greyscale correction, underestimations of the areas of leaves with chlorophyll deficiencies could be avoided. There are two possible reasons for the second observation. It was noticed that it was not possible to make all leaves lie flat on the scanner bed because of their rigidity and non-planarity. This would have caused some small portions in the ensuing images to be possibly eclipsed resulting in an underestimation of the areas by the DIA. The same cautions have been indicated by other researchers (Rico-Garcia et al., 2009; Tsuda, 1999). On the other hand, while sampling leaf surfaces for the extraction of the Hughes constant value in

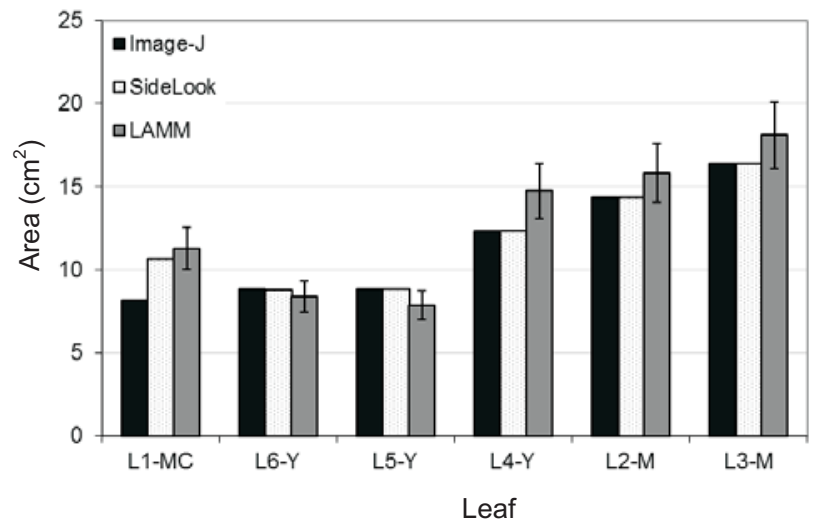

Fig. 3. Comparison of the areas of the 6 selected leaves, L1 to L6, of the plant $M$. splendens obtained by LAMM using the $\mathrm{K}_{\mathrm{G}}$ value (Table 1) and the DIA techniques using SL and IJ. The overall RD of the LAMM with respect to the SL is $10.37 \pm 5.69 \%$. The Student t-test indicated no statistically significant difference between the LAMM and DIA values of the overall average of the areas.

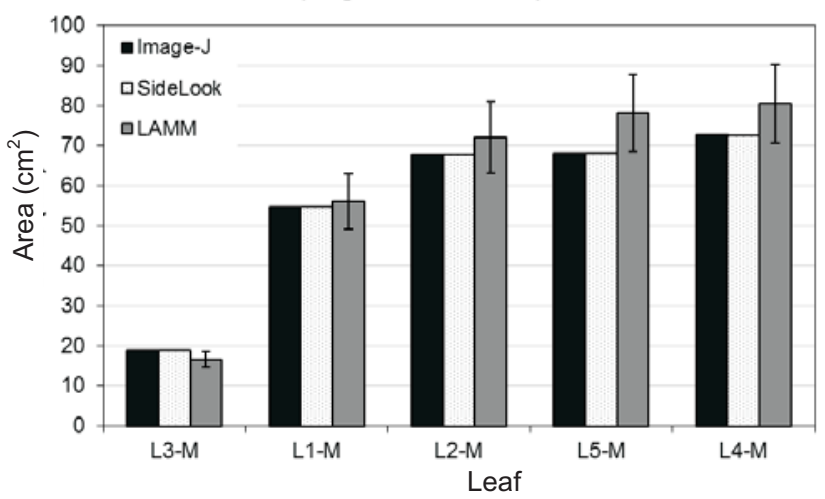

Fig. 4. Comparison of the areas of the 5 mature leaves of $S$. wallisii obtained by LAMM using $\mathrm{K}_{\mathrm{G}}$ (Table 1) and the DIA using SL and IJ. The overall RD of the LAMM with respect to the SL is $9.25 \pm 4.90 \%$. The differences in the overall average values of the areas obtained by the LAMM and by the DIA, were not statistically significant.

the LAMM method, the tendency - albeit unintentional - is to under-include the ribbed zones where the leaf thickness $\tau$ is slightly higher. The lack of a thorough representation of these regions could mean that the $\mathrm{K}_{\mathrm{G}}$ value tends to shift slightly higher, resulting in a small increase of the area. An automation of the LAMM technique would help to improve the randomization of the sampling. In anycase, this effect is likely to be small and, as seen for the case of the geometrical discs extracted from leaves, the intrinsic accuracy of the LAMM technique is high.

Thus for real leaves the LAMM method produces values not very different from the DIA and with accuracies of the same numerical magnitude. 


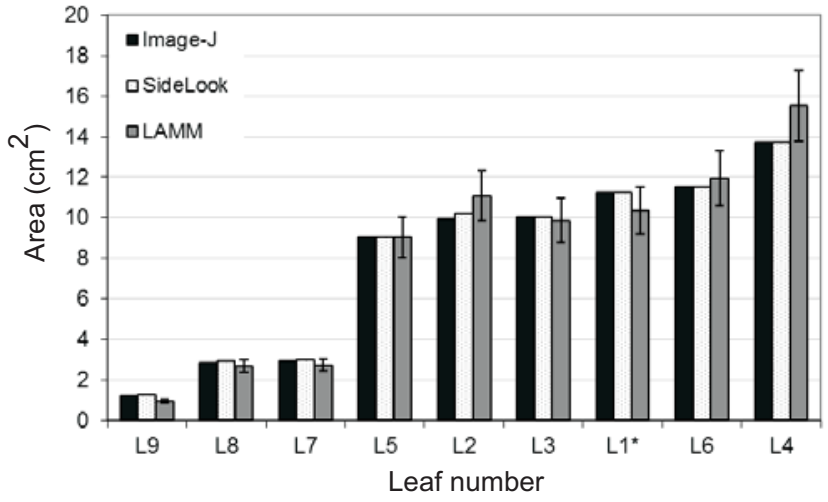

Fig. 5. Foliar areas of 9 leaves of the plant M. splendens, L1 to L9, selected at random and measured by the techniques of DIA (IJ and SL) and by LAMM using the $\mathrm{K}_{\mathrm{G}}$ value obtained from the leaves of a different plant of the same species (Table 1). L1 was a maturechlorotic leaf (indicated as L1*). The average RD of the LAMM with respect to the SL measurements was $8.50 \pm 6.90 \%$. The t-test between the LAMM and DIA values of the average area for the entire ensemble of leaves showed no statistically significant difference.

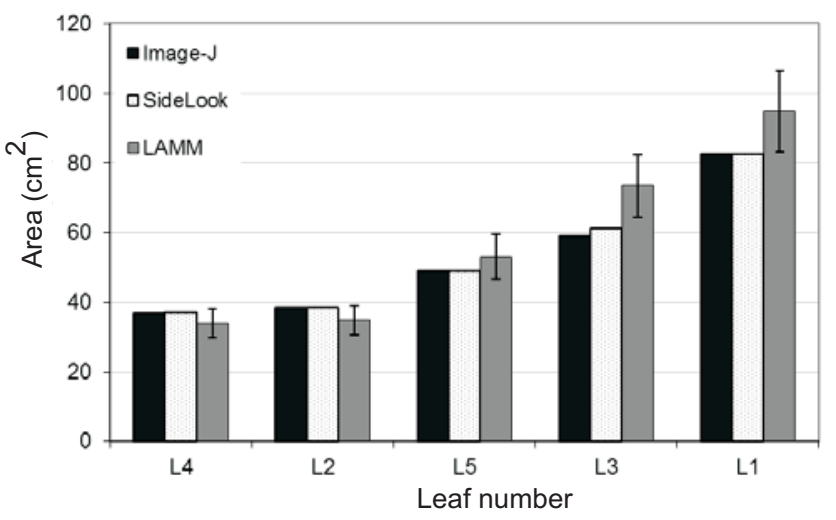

Fig. 6. Foliar areas of 5 leaves of the plant $S$. wallisii selected at random and measured by the techniques of DIA (IJ and SL) and by LAMM using the $\mathrm{K}_{\mathrm{G}}$ value obtained from a set of leaves of a different plant of the same species (Table 1). The average RD of the LAMM with respect to the DIA measurements was $12.08 \pm 5.43 \%$. The t-test between the LAMM and DIA values of the overall average area showed no statistically significant difference.

Figure 5 shows the LAMM and DIA measured areas of 9 leaves of a different $M$. splendens plant that also included one mature-chlorotic leaf. For the LAMM measurement, the $\mathrm{K}_{\mathrm{G}}$ value obtained from the first (and therefore different) M. splendens plant was used. Figure 6 shows the analogous measurements for the 5 leaves of a different $S$. wallisii plant where again the $\mathrm{K}_{\mathrm{G}}$ value from the first $S$. wallisii had been used. The t-test comparison between the overall average areas obtained by the LAMM and by the DIA in the case of both plants revealed that there was no statistically significant difference $(p<0.05)$ between the two types of measurement, just as in the case of the intra-plant measurements stated in the preceding where the intra-plant $\mathrm{K}_{\mathrm{G}}$ value had been used. Further, the overall average RD between the LAMM and the DIA (SIDELOOK) were $8.5 \pm 6.9 \%$ and $12.1 \pm 5.4 \%$ for the M. splendens and $S$. wallisii, respectively. A comparison between these RD values with those for the two plants of the same species of the first set revealed that there was no statistically significant difference $(p<0.05)$ between them. Since the DIA measurements are independently performed on each plant (with no carry-over values such as the $\mathrm{K}_{\mathrm{G}}$ ), the similitude of the $\%$ average relative deviations for the two sets of the $M$. splendens and $S$. wallisii plants implies that the LAMM for the second set using the $\mathrm{K}_{\mathrm{G}}$ derived from the first set, produces results that are similar in the level of accuracy. This means that the $\mathrm{K}_{\mathrm{G}}$ value can be exported from one plant to another of the same species with little if any consequent loss in the degree of accuracy of the LAMM measurements. This exportability is the direct consequence of the conservation of the Hughes constant within a species (Roderick and Cochrane, 2002). The implication of this finding is that the LAMM method reduces to a very simple operation when the foliar area of a group of plants of the same species located in a stand is desired. The $\mathrm{K}_{\mathrm{G}}$ values can be extracted from a representative plant and exported to the others. In the interest of a homogeneous representation however, it would be preferable to obtain the $\mathrm{K}_{\mathrm{G}}$ value as the average of the $\mathrm{K}$ values of leaves excised from various plants of the mono-specific set. For groups of mixed species, the process is replicated for each species-distinguished group.

The degree of linear correlation between the DIA methods based on both SIDELOOK and IMAGE-J and the method of LAMM for all cases concerning leaves studied in this work, is shown in Fig. 7. As can be seen from the result $\mathrm{R}^{2}=0.99$, the correlation is excellent.

A number of previous workers (Ma et al., 1992; Sharatt and Baker, 1986; Vile et al., 2005) have utilised the concept of the SLA, leaf dry mass (LDM) and the dimensional attributes of leaves to obtain the quantification of a particular desired attribute. In the works of Sharatt and Baker (1986) and Ma et al. (1992), the leaf area (LA) is connected to the LDM via a regression fitted equation, while in the work of Vile et al. (2005) the leaf thickness $(\tau)$ is connected to the inverse of the product of SLA and LDM by a regression equation whose coefficient they show is the inverse of the fresh leaf density $\left(\rho^{-1}\right)$. These methods come under the purview of the general gravimetric method discussed by Jonckheere et al. (2004). However, they are all relative methods. They need a prior calibration via the exact measurement of the very attribute they seek to measure (LA or $\tau$ ) in the given plant species in order to obtain the fit coefficients. Strictly, from the standpoint of measurement methodology, such a cyclic argument is of limited utility. In contrast, the LAMM is an absolute method requiring no prior calibration fits. It measures the Hughes constant for the given species in 


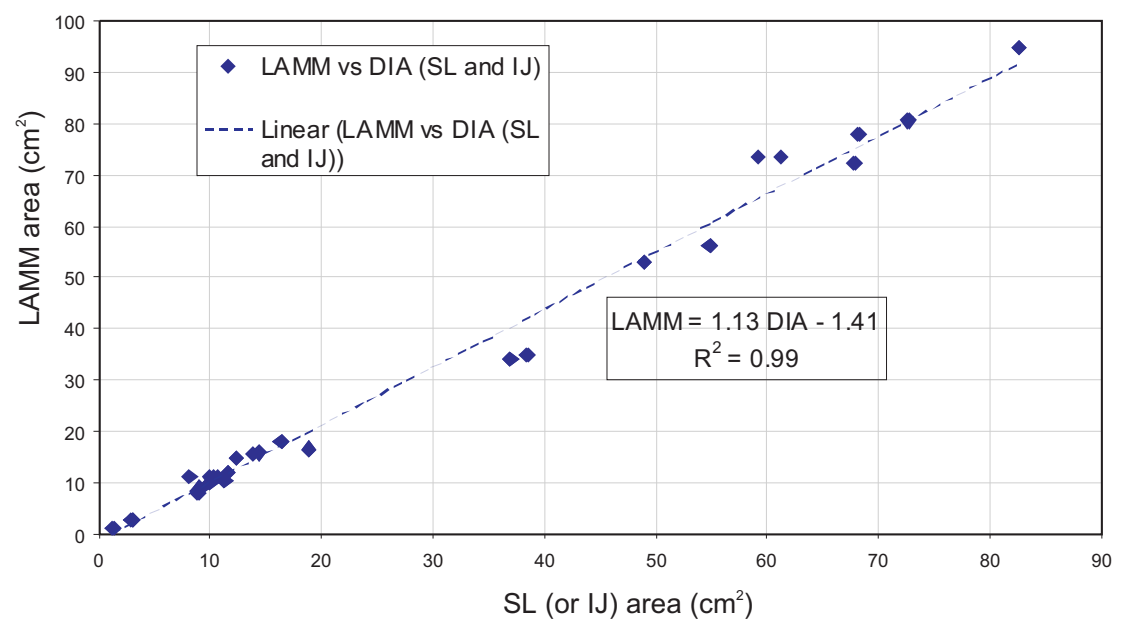

Fig. 7. Linear correlation between the LAMM and the DIA measurements using Sidelook and Image-J, of the leaf areas of the plants M. splendens and S. wallisii.

situ. It can also be fine-tuned to any desired level of stratification. The combination of the conservativeness of the Hughes constant and the idea of methodology borrowed from thick-film technology permits the LAMM to be an absolute technique. By doing away with the calibration and drying steps in the gravimetric technique, LAMM substantially improves on the speed of operation. Thus LAMM may find utility in those cases where an alternative to the DIA without the disadvantages of the gravimetric method, is sought (Jonckheere et al., 2004).

The Hughes constant $\mathrm{K}$ is the pivot in the LAMM method. As is true for other biometric indices, $\mathrm{K}$ may change with different environmental conditions for the given species. Since the LAMM method measures the K value in situ for the given species, the variability of $\mathrm{K}$ under different environmental conditions becomes an irrelevant issue.

Some other salient features of LAMM are its economy and ease of operation, both of which are important for small agribusinesses particularly in developing countries. In the future LAMM could be automated and its procedure improved with the use of high-quality die-punches of accurate IDs and with an ID range that extends to much lower sizes so that the areas of finely pinnated leaves may also be measured. Furthermore, the method does not interfere with other optical methods of foliar analysis and could be added on as an additional feature to existing equipment.

\section{CONCLUSIONS}

1. Leaf-area-measurement-by-mass is a simple, accurate, rapid and economic technique for the measurement of the foliar area of herbaceous plants.

2. While this work illustrates the application of leafarea-measurement-by-mass to broad-leaved species, the principle underlying it is universal and applicable to the leaves of all plants, as long as the conservativeness of the Hughes constant for the species is not destroyed.

\section{REFERENCES}

Behera S.K., Srivastava P., Pathre U.V., and Tuli R., 2010. An indirect method of estimating leaf area index in Jatropha curcas L. using LAI-2000 Plant Canopy Analyzer. Agric. Forest Meteor., 150, 307-311.

Coombs J., Hall D.O., Long S.P., and Scurlock J.M.O., 1985. Techniques in bioproductivity and photosynthesis, Pergamon Press, Oxford, UK.

Hughes A.P., Cockshull K.E., and Heath O.V.S., 1970. Leaf area and absolute leaf water content. Annals Botany, 34, 259-265.

Igathinathane C., Prakash V.S.S., Padma U., Ravi Babu G., and Womac A.R., 2006. Interactive computer software development for leaf area measurement. Computers Electronics Agric., 51, 1-16.

Jonckheere I., Fleck S., Nackaerts K., Muys B., Coppin P., Weiss M., and Baret F., 2004. Review of methods for in situ leaf area index determination: Part I. Theories, sensors and hemispherical photography. Agric. Forest Meteor., 121, 19-35.

Kirk K., Andersen H.J., Thomsen A.G., Jorgensen J.R., and Jorgensen R.N., 2009. Estimation of leaf area index in cereal crops using red-green images. Biosys. Eng., 104, 308-317.

Korva J.T. and Forbes G.A., 1997. A simple and low-cost method for leaf area measurement of detached leaves. Exp. Agric., 33, 65-72.

Ma L., Gardner F.P., and Selamat A., 1992. Estimation of leaf area from leaf and total mass measurements in peanut. Crop Sci., 32, 467-471.

Mandel J., 1984. The statistical analysis of experimental data. Dover Publications Inc., New York, USA.

Meziane D. and Shipley B., 1999. Interacting determinants of specific leaf area in 22 herbaceous species: effects of irradiance and nutrient availability. Plant Cell Environ., 22, 447-459.

Mohsenin N.N., 1986. Physical properties of plant and animal materials. Gordon Breach Sci. Press, New York, USA.

Nobis M. and Hunziker U., 2005. Automatic thresholding for hemispherical canopy-photographs based on edge detection. Agric. Forest Meteor., 128, 243-250. 
O'Neal M., Landis D.A., and Isaacs R., 2002. An inexpensive, accurate method for measuring leaf area and defoliation through digital image analysis. J. Economic Entomology, 95, 1190-1194.

Rico-García E., Hernández-Hernández F., Soto-Zarazúa G., and Herrera-Ruiz G., 2009. Two new methods for the estimation of leaf area using digital photography. Int. J. Agric. Biolog., 11, 397-400.

Roderick M.L. and Cochrane M.J., 2002. On the conservative nature of the leaf mass-area relationship. Annals Botany, 89, 537-542.

Sharatt B.S. and Baker D.G., 1986. Alfalfa leaf area as a function of dry matter. Crop Sci., 26, 1040-1043.
Singh M., Saxena N.P., and Saxena M.C., 1995. Estimation of leaf pinnule area in chickpea leaves: an errors-in-variables approach. J. Appl. Statistics, 22, 37-43.

Tsuda M., 1999. Errors in leaf Area measurement with an automatic Area meter due to leaf chlorophyll in crop plants. Annals Botany, 84, 799-801.

Vile D., Garnier E., Shipley B., Laurent G., Navas M.-L., Roumet C., Lavorel S., Díaz S., Hodgson J.G., Lloret F., Midgley G.F., Porter H., Rutherford M.C., Wilson P.J., and Wright I.J., 2005. Specific leaf area and dry matter content estimate thickness in laminar leaves. Annals Botany, 96, 1129-1136.

Zehm A., Nobis M., and Schwabe A., 2003. Multiparameter analysis of vertical vegetation structure based on digital image processing. Flora, 198, 42-160. 